\title{
ARBORIZAÇÃO DE RUAS E PRAÇAS EM SALVADOR, BAHIA
}

\author{
Gabriel Salles Góes; Maria Zélia Alencar de Oliveira² \\ (recebido em 30.09.2010 e aceito para publicação em 08.07.2011)
}

\section{RESUMO}

Este trabalho teve por objetivo avaliar a comunidade arbórea de ruas e praças em Salvador, BA, com base em inventários quantitativos da arborização, registrados em relatórios técnicos e publicações científicas. Analisou-se a riqueza, diversidade, abundância de espécies exóticas, nativas não regionais e nativas regionais, freqüência de espécies que fornecem alimento à fauna e ocorrência de espécies tóxicas, alergênicas e invasoras. Foram amostrados 2.469 indivíduos, pertencentes a 61 gêneros e 27 famílias, com predomínio de Fabaceae. Verificou-se uma grande riqueza de espécies $(R=82)$, com dominância de amendoeiras (Terminalia catappa), sombreiros (Clitoria fairchildiana) e mongubas (Pachira aquatica), que juntas representam $28,4 \%$ da comunidade. Cabe ressaltar a predominância de indivíduos de espécies exóticas $(53,3 \%)$ e os poucos representantes de espécies nativas do domínio da Mata Atlântica baiana $(17,1 \%)$. Houve um baixo percentual de espécies que ofereciam alimento à fauna $(26,4 \%)$ e ocorreram seis espécies tóxicas e alergênicas, entre elas a espirradeira (Nerium oleander) e chapéu-de-Napoleão (Thevetia peruviana). Foram identificadas 10 espécies potencialmente invasoras, com maior abundância de: amendoeiras (T. catappa), casuarinas (Casuarina equisetifolia) e sabiás (Mimosa caesalpiniifolia).

Palavras-chave: Biodiversidade; Exóticas; Nativas; Tóxicas; Invasoras.

1 Biólogo, Mestrando em Produção Vegetal, UESC, Ilhéus, BA, gsgoes@gmail.com; 2 Mestre em Fitopatologia, pesquisadora FAPESB/EBDA, Salvador, Ba, zeliaao@gmail.com 


\section{URBAN FOREST OF STREETS AND SQUARES IN SALVADOR, BA}

\section{ABSTRACT}

This study aimed evaluate trees of streets and squares in Salvador, BA, based on quantitative urban forest inventories, registered in technical reports and scientific publications. The following aspects were analyzed: species richness and diversity, abundance of exotics, non-regionals and regionals native species, frequence of species which provide food to fauna, and the occurrence of toxic, allergenic, and invasive species. 2,469 individuals were sampled, belonging to 61 genera and 27 botanical families, with predominance of Fabaceae. A high species richness was observed $(R=82)$, with dominance of Terminalia catappa, Clitoria fairchildiana, and Pachira aquatica, which, together, represent $28.4 \%$ of tree community. It is worth mentioning the predominance of individuals belonging to exotic species (53.3\%) and few individuals of native species of Bahia Atlantic Forest (17.1\%). There was a low percentage of species which provided food to fauna (26.4\%) and six toxic and allergenic species were found, being among them Nerium oleander and Thevetia peruviana. Ten potentially invasive species were identified with higher abundance of $T$. catappa, Casuarina equisetifolia, and Mimosa caesalpiniifolia.

Keywords: Biodiversity; Exotic species; Native species; Toxic species; Invasive species.

\section{INTRODUÇÃO}

São muitos os benefícios proporcionados por uma arborização planejada e eficiente. As árvores nas ruas e praças melhoram o microclima e o conforto térmico, através da redução da incidência direta da radiação solar (Spangenberg et al., 2008; Herrmann, 2008). A menor temperatura das construções contribui para redução dos gastos com refrigeração e ventilação (Velasco, 2007). O resfriamento do ar pelas árvores diminui a produção fotoquímica de ozônio, além de adsorver poluentes e material particulado (Taha et al., 1997).

A vegetação ao longo das estradas traz maior conforto visual e redução de elementos que possam distrair os motoristas (Wolf, 2003). A sombra das árvores contribui para a melhor perfomance do pavimento das ruas, reduzindo os custos de manutenção 
(McPherson e Muchnick, 2005). Nas ruas, os indivíduos arbóreos interceptam a água da chuva, amenizando o escoamento que causa alagamentos (Silva, 2008).

Além destes benefícios, Pivetta e Silva Filho (2002) citam a redução da poluição sonora, o melhor efeito estético e maior bem-estar psicológico, os quais contribuem para a melhoria da qualidade de vida e para o restabelecimento da relação com o meio natural.

Os "corredores verdes" nas cidades contribuem para a conservação da biodiversidade (Bryant, 2006). Plantas raras podem ser cultivadas para manutenção de um banco genético (Savard; Clergeau; Mennechez, 2000). As árvores nas vias garantem abrigo e diversificação de fontes de alimentação para a fauna (Brun; Link; Brun, 2007). Pivetta e Silva Filho (2002) argumentam que a questão de frutos para consumo humano é assunto polêmico, alguns acreditam estimular a depredação e outros contestam que se deve lutar pela concientização da população.

Apesar de tantos benefícios, a arborização viária é negligenciada pelo poder público. Em Salvador, BA, as poucas Leis que tratam de árvores urbanas são: a Lei no 5.493 de 19 de Janeiro de 1999, que dispõe sobre a conservação, preservação, poda, agressão, erradicação e a reposição de árvores no município e dá outras providências (Prefeitura Municipal de Salvador, 2009); e a Lei no 4.456 de 09 de Dezembro de 1991, que obriga o plantio de árvores frutíferas nas avenidas de vale, bem como nas avenidas que disponham de áreas marginais favoráveis para tal (Prefeitura Municipal de Salvador, 2009).

Não existe um Plano Diretor de Arborização para a capital baiana e escassos são os estudos de suas árvores. Oliveira et al. (2007) investigaram algumas espécies vegetais nativas, com potencial paisagístico, para arborização de áreas verdes de Salvador. Outro estudo foi realizado por Oliveira (2007) em relação às pragas e fungos patogênicos associados à vegetação da cidade.

Carvalho, Roque e Guedes (2007) realizaram um levantamento da arborização de espaços livres dos campi da Universidade Federal da Bahia. Maliarenko e Brito (1996) estudaram uma proposta de intervenção para o trecho Pituba - Costa Azul, da Orla de Salvador, utilizando-se de espécies nativas de restinga.

Este trabalho, de conclusão de curso do primeiro autor, teve por objetivo geral, estudar a comunidade arbórea de ruas e praças em Salvador-BA, com base em inventários quantitativos da arborização, para analisar a riqueza e diversidade da comunidade arbórea; a abundância de espécies exóticas, nativas não regionais e nativas regionais; a freqüência de espécies que forneçam alimento à fauna (incluso o homem); e detectar a ocorrência de espécies tóxicas, alergênicas e invasoras.

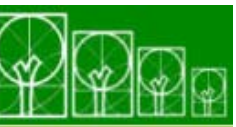

S $\cdot \mathbf{B} \cdot \mathbf{A} \cdot \mathbf{U}$ Soc. Bras. de Arborização Urbana 


\section{MATERIAIS E MÉTODOS}

\section{Área de estudo}

O município de Salvador é a capital do estado da Bahia (BA) e sua região metropolitana (RMS) é composta de dez municípios, cuja área total é de $2.186,61 \mathrm{~km}^{2}$ e a população, em 2001, era de 3.021.572 habitantes (CONDER, 2006). Localiza-se dentro do domínio do Bioma Mata Atlântica (MMA, 2002).

A temperatura média anual é de $24^{\circ} \mathrm{C}$ a $26^{\circ} \mathrm{C}$, sendo que a máxima nunca ultrapassa $36^{\circ} \mathrm{C}$. Os meses de temperatura mais amena são, geralmente, junho e julho, sendo que a média do mês mais frio varia de $20^{\circ}$ a $24^{\circ} \mathrm{C}$ (IBGE, 1977). Os índices pluviométricos anuais estão entre 1750 a 2000 mm. O máximo de pluviosidade se dá no Outono (mais freqüentemente) ou no Inverno, sendo o trimestre mais chuvoso os meses de abril-maiojunho. O mínimo se dá na primavera ou verão, mas não há estação seca (IBGE, 1977).

\section{Materiais e métodos}

A busca por inventários quantitativos, com a frequência de indivíduos por espécie, foi efetuada em periódicos e publicações da área como a Revista da Sociedade Brasileira de Arborização Urbana, Revista Brasileira de Botânica, resumos de congressos, entre outros, e em relatórios técnicos disponíveis nos órgãos municipais responsáveis pela arborização e áreas verdes (SMA - Superintendência de Meio Ambiente; SUCOP - Superintendência de Conservação e Obras Públicas; Jardim Botânico de Salvador e no Parque Joventino Silva).

Os nomes científicos foram adotados conforme TROPICOS (2009), sendo feitas, quando possíveis, as devidas correções. Subespécies e variedades foram consideradas em nível de espécie. A classificação das famílias segue APG II (2003), como adotado por Souza e Lorenzi (2005).

Foram excluídas da análise as espécies não-arbóreas, como arbustos, palmeiras (Arecaceae), entre outras. Apesar de sua importância paisagística e ecológica, estas não proporcionam tantos benefícios quanto às árvores, devido ao reduzido tamanho da copa e por prejudicar o trânsito de pedestres (no caso de arbustos e touceiras).

A classificação quanto à origem dividiu as espécies em três grupos: exóticas do Brasil (EX); nativas do Brasil, mas não regionais (BR); e aquelas nativas regionais do Domínio da Mata Atlântica no estado da Bahia (NR). Esta classificação é parecida à adotada por Isernhagen, Bourlegat e Carboni (2009). 
A origem das espécies e aquelas fornecedoras de alimento à fauna, foram levantadas com base em Lorenzi (2002), Lorenzi et al. (2003; 2006). Quanto ao provimento de alimento, foram consideradas tanto aquelas espécies que fornecem alimento somente à fauna, quanto aquelas com produtos comestíveis para o homem.

As espécies invasoras foram levantadas com base no estudo "Contextualização sobre espécies exóticas invasoras - Dossiê Pernambuco" (Almeida et al., 2009), levando-se em conta a proximidade geográfica deste estado. As espécies tóxicas e com princípios alergênicos foram levantadas a partir do SINITOX (2009), Lorenzi (2002), Lorenzi et al. (2003, 2006), Lorenzi e Souza (2001).

\section{RESULTADOS E DISCUSSÃO}

Foram encontrados os seguintes relatórios técnicos e estudos com inventários da arborização:

1. "SPJ - Corredor da Centenário" - inventário quali-quantitativo das árvores presentes nas avenidas Centenário e Vale dos Barris, ruas Airosa Galvão, Doutor Artur Neiva, Plínio Moscoso, Deocleciano Barreto, Martagão Gesteira, Engenheiro Celso Torres, Beirute, Doutor Arlindo de Assis e praças do IBIT, dos Reis Católicos e Doutor João Mangabeira (Chão Verde, 2002);

2. "Vistoria da Arborização do Dique do Tororó" - relação de indivíduos arbóreos e palmeiras que circundam o Dique, com uma avaliação do estado fitossanitário e recomendações de manejo (Oliveira; Nakagawa; Figueiredo, 2000);

3. "Diagnóstico da vegetação da Rua Miguel Calmon" - inventário quali-quantitativo das árvores da referida rua (Longa; Barbosa; Oliveira, 1998);

4. "Caracterização de vias de circulação. Avenida Antonio Carlos Magalhães - Itaigara" - relação das espécies vegetais e suas freqüências na área especificada (SPJ, 2008a);

5. "Caracterização de vias de circulação. Avenida Juracy Magalhães Júnior" (SPJ, 2007);

6. "Caracterização de vias de circulação. Avenida Vasco da Gama" (SPJ, 2008b);

7. "Levantamento das espécies arbóreas da Universidade Federal da Bahia, Salvador, Bahia" - feito por Carvalho, Roque e Guedes (2007) nos campi da UFBA dos bairros de Ondina, Federação, São Lázaro e Canela. 
Pelo fato destas áreas localizarem-se na Macroárea Lucaia/Costeira (CONDER, 2006), essa amostragem será assim denominada. Contudo, é importante ressaltar um fato. Os resultados obtidos a partir dos estudos de Carvalho, Roque e Guedes (2007) na Universidade Federal da Bahia, em comparação com as outras áreas de Salvador, demonstram que esta apresenta uma arborização bastante diferenciada, pelos seguintes motivos:

- 51 espécies ocorreram exclusivamente na UFBA, sendo que o número de espécies exclusivas de outras áreas é de oito, na Av. Vasco da Gama, quatro, nas Avenidas Centenário, Juracy Magalhães Jr. e ACM, e uma no Dique do Tororó;

- A riqueza encontrada na UFBA (103 espécies) é maior que aquela encontrada nas outras áreas juntas (82 espécies), mesmo considerando o total de indivíduos amostrados, 1723 e 2469 respectivamente.

- A freqüência de espécies nativas regionais é bem maior na UFBA que nas outras áreas (à exceção da Av. Juracy Magalhães Jr.).

Por esses aspectos e pelo fato da arborização da UFBA estar historicamente desvinculada da intervenção da Prefeitura Municipal de Salvador, entende-se que esta não pode ser analisada juntamente com as outras áreas. Então, far-se-á a análise em conjunto das outras seis áreas amostradas (Corredor da Centenário, Dique do Tororó, R. Miguel Calmon, Avenidas ACM, Juracy Magalhães Jr. e Vasco da Gama). Vale ressaltar que as avenidas supracitadas são parkways com canteiros largos.

Ao todo, foram amostrados 2469 indivíduos, pertencentes a 82 espécies, 61 gêneros, dispostos em 27 famílias. A relação destas e suas abundâncias encontram-se na Tabela 1.

Tabela 1 - Espécies arbóreas encontradas na Macroárea Lucaia/Costeira ( $P$ - Abundância total, $\mathrm{Pi}$ - Abundância relativa).

Table 1 - Tree species found in Lucaia/Costeira Macroarea ( $\mathrm{P}$ - Total abundance, Pi Relative abundance).

\begin{tabular}{|c|c|c|c|c|c|c|}
\hline $\mathrm{N}^{\mathbf{0}}$ & Espécies & & Família & Nome popular & $\mathbf{P}$ & $\mathrm{Pi}(\%)$ \\
\hline 1 & \multicolumn{2}{|l|}{ Terminalia catappa L. } & Combretaceae & Amendoeira & 266 & 10,80 \\
\hline 2 & $\begin{array}{l}\text { Clitoria fairchildiana } \\
\text { Howard }\end{array}$ & R.A. & Fabaceae & Sombreiro & 239 & 9,68 \\
\hline 3 & Pachira aquatica Aubl. & & Malvaceae & Monguba & 195 & 7,90 \\
\hline 4 & $\begin{array}{l}\text { Tabebuia pentaphylla } \\
\text { Hemsl. }\end{array}$ & (L.) & Bignoniaceae & Ipê-bálsamo & 136 & 5,51 \\
\hline 5 & Delonix regia (Bojer) Raf. & & Fabaceae & Flamboyant & 132 & 5,35 \\
\hline 6 & Bauhinia variegata $\mathrm{L}$. & & Fabaceae & Pata-de-vaca-rosa & 119 & 4,82 \\
\hline
\end{tabular}




\begin{tabular}{|c|c|c|c|c|c|}
\hline $\mathbf{N}^{\mathbf{0}}$ & Espécies & Família & Nome popular & $\mathbf{P}$ & Pi (\%) \\
\hline 7 & $\begin{array}{l}\text { Caesalpinia peltophoroides } \\
\text { Benth. }\end{array}$ & Fabaceae & Sibipiruna & 111 & 4,50 \\
\hline 8 & Albizia lebbeck (L.) Benth. & Fabaceae & Língua-de-sogra & 106 & 4,29 \\
\hline 9 & Lagerstroemia indica L. & Lythraceae & Resedá & 92 & 3,73 \\
\hline 10 & Cassia fistula $\mathrm{L}$. & Fabaceae & Chuva-de-ouro & 86 & 3,48 \\
\hline 11 & Ficus benjamina L. & Moraceae & $\begin{array}{l}\text { Figueira- } \\
\text { benjamina }\end{array}$ & 82 & 3,32 \\
\hline 12 & $\begin{array}{l}\text { Handroanthus avellanedae } \\
\text { Lorentz ex Griseb. }\end{array}$ & Bignoniaceae & Ipê-rosa & 75 & 3,04 \\
\hline 13 & Ceiba pentandra (L.) Gaertn. & Malvaceae & Sumaúma & 65 & 2,63 \\
\hline 14 & Caesalpinia ferrea Mart. & Fabaceae & Pau-ferro & 62 & 2,51 \\
\hline 15 & $\begin{array}{l}\text { Filicium decipiens (Wight \& } \\
\text { Arn.) Thwaites }\end{array}$ & Sapindaceae & $\begin{array}{l}\text { Árvore- } \\
\text { samambaia }\end{array}$ & 48 & 1,94 \\
\hline 16 & Mangifera indica L. & Anacardiaceae & Mangueira & 43 & 1,74 \\
\hline 17 & $\begin{array}{l}\text { Handroanthus chrysotrichus } \\
\text { (Mart. ex A. DC.) Standl. }\end{array}$ & Bignoniaceae & Ipê-amarelo & 40 & 1,62 \\
\hline 18 & Caesalpinia echinata Lam. & Fabaceae & Pau-Brasil & 39 & 1,58 \\
\hline 19 & $\begin{array}{l}\text { Senna siamea (Lam.) H.S. Irwin } \\
\& \text { Barneby }\end{array}$ & Fabaceae & Cássia-siamesa & 35 & 1,42 \\
\hline 20 & $\begin{array}{l}\text { Handroanthus caraiba (Mart.) } \\
\text { Bureau }\end{array}$ & Bignoniaceae & Caraibeira & 35 & 1,42 \\
\hline 21 & Casuarina equisetifolia L. & Casuarinaceae & Casuarina & 34 & 1,38 \\
\hline 22 & Triplaris pachau Mart. & Polygonaceae & Triplaris & 29 & 1,17 \\
\hline 23 & Adenanthera pavonina L. & Fabaceae & Carolina & 24 & 0,97 \\
\hline 24 & Ficus elastica Roxb. & Moraceae & $\begin{array}{l}\text { Seringueira-de- } \\
\text { jardim }\end{array}$ & 22 & 0,89 \\
\hline 25 & Jacaranda mimosifolia D. Don & Bignoniaceae & Ipê-roxo & 20 & 0,81 \\
\hline 26 & Spondias monbim L. & Anacardiaceae & Cajazeira & 19 & 0,77 \\
\hline 27 & Cecropia pachystachya Trécul & Cecropiaceae & Embaúba & 19 & 0,77 \\
\hline 28 & $\begin{array}{l}\text { Peltophorum dubium (Spreng.) } \\
\text { Taub. }\end{array}$ & Fabaceae & Canafístula & 18 & 0,73 \\
\hline 29 & $\begin{array}{l}\text { Licania tomentosa (Benth.) } \\
\text { Fritsch }\end{array}$ & Chrysobalanaceae & Oiti-mirim & 17 & 0,69 \\
\hline 30 & Cassia grandis L. f. & Fabaceae & Cássia-grande & 16 & 0,65 \\
\hline 31 & $\begin{array}{l}\text { Tabebuia } \\
\text { Sandwith }\end{array}$ & Bignoniaceae & Ipê-branco & 16 & 0,65 \\
\hline 32 & Cassia javanica L. & Fabaceae & Cássia-javanesa & 13 & 0,53 \\
\hline 33 & Mimosa caesalpiniifolia Benth. & Fabaceae & Sabiá & 12 & 0,49 \\
\hline 34 & Syzygium cumini (L.) Skeels & Myrtaceae & Jamelão & 11 & 0,45 \\
\hline 35 & $\begin{array}{l}\text { Senna spectabilis (DC.) H.S. } \\
\text { Irwin \& Barneby }\end{array}$ & Fabaceae & São-João & 11 & 0,45 \\
\hline 36 & Persea americana Mill. & Lauraceae & Abacateiro & 10 & 0,41 \\
\hline 37 & Prosopis ruscifolia Griseb. & Fabaceae & Algarobo & 10 & 0,41 \\
\hline 38 & Schinus terebinthifolia Raddi & Anacardiaceae & Aroeira & 9 & 0,36 \\
\hline 39 & $\begin{array}{l}\text { Tibouchina granulosa (Desr.) } \\
\text { Cogn. }\end{array}$ & Melastomataceae & Quaresmeira & 9 & 0,36 \\
\hline 40 & Acacia farnesiana (L.) Willd. & Fabaceae & $\begin{array}{l}\text { Vinhático-de- } \\
\text { espinho }\end{array}$ & 8 & 0,32 \\
\hline 41 & Ficus guaranítica Chodat & Moraceae & Figueira-branca & 8 & 0,32 \\
\hline 42 & Anacardium occidentale L. & Anacardiaceae & Cajueiro & 7 & 0,28 \\
\hline 43 & Tipuana tipu (Benth.) Kuntze & Fabaceae & Tipuana & 7 & 0,28 \\
\hline
\end{tabular}




\begin{tabular}{|c|c|c|c|c|c|}
\hline $\mathbf{N}^{\mathbf{0}}$ & Espécies & Família & Nome popular & $\mathbf{P}$ & Pi (\%) \\
\hline 44 & Triplaris gardneriana Wedd. & Polygonaceae & Pajaú & 7 & 0,28 \\
\hline 45 & $\begin{array}{l}\text { Pithecellobium diversifolium } \\
\text { Benth. }\end{array}$ & Fabaceae & - & 6 & 0,24 \\
\hline 46 & Simarouba amara Aubl. & Simaroubaceae & Pau-paraíba & 6 & 0,24 \\
\hline 47 & Byrsonima stipulacea A. Juss. & Malpighiaceae & Murici-do-litoral & 6 & 0,24 \\
\hline 48 & $\begin{array}{l}\text { Leucaena leucocephala (Lam.) } \\
\text { de Wit }\end{array}$ & Fabaceae & Leucena & 5 & 0,20 \\
\hline 49 & Artocarpus heterophyllus Lam. & Moraceae & Jaqueira & 5 & 0,20 \\
\hline 50 & Genipa americana L. & Rubiaceae & Jenipapo & 5 & 0,20 \\
\hline 51 & Hovenia dulcis Thunb. & Rhamnaceae & Uva-japonesa & 5 & 0,20 \\
\hline 52 & $\begin{array}{l}\text { Sterculia striata A. St.-Hil. \& } \\
\text { Naudin }\end{array}$ & Malvaceae & Chichá-do-cerrado & 5 & 0,20 \\
\hline 53 & Tamarindus indica L. & Fabaceae & Tamarindeiro & 4 & 0,16 \\
\hline 54 & $\begin{array}{l}\text { Samanea tubulosa (Benth.) } \\
\text { Barneby \& J.W. Grimes }\end{array}$ & Fabaceae & Alfarobo & 4 & 0,16 \\
\hline 55 & Parkinsonia aculeata L. & Fabaceae & Turco & 4 & 0,16 \\
\hline 56 & Psidium guajava L. & Myrtaceae & Goiabeira & 3 & 0,12 \\
\hline 57 & $\begin{array}{l}\text { Pithecellobium diversifolium } \\
\text { Benth. }\end{array}$ & Fabaceae & - & 3 & 0,12 \\
\hline 58 & Salix babylonica L. & Salicaceae & Chorão & 3 & 0,12 \\
\hline 59 & Psidium cattleianum Sabine & Myrtaceae & Araçá & 3 & 0,12 \\
\hline 60 & Ficus lyrata Warb. & Moraceae & Figueira-lira & 3 & 0,12 \\
\hline 61 & $\begin{array}{l}\text { Dalbergia nigra (Vell.) Allemao } \\
\text { ex Benth. }\end{array}$ & Fabaceae & $\begin{array}{l}\text { Jacarandá-da- } \\
\text { Bahia }\end{array}$ & 3 & 0,12 \\
\hline 62 & $\begin{array}{l}\text { Schizolobium parahyba (Vell.) } \\
\text { S. F. Blake }\end{array}$ & Fabaceae & Guapuruvu & 3 & 0,12 \\
\hline 63 & Guarea guidonia (L.) Sleumer & Meliaceae & Bilreiro & 2 & 0,08 \\
\hline 64 & Guazuma ulmifolia Lam. & Malvaceae & Embira & 2 & 0,08 \\
\hline 65 & Triplaris brasiliana Cham. & Polygonaceae & - & 2 & 0,08 \\
\hline 66 & Zanthoxylum rhoifolium Lam. & Rutaceae & Mamiqueira & 2 & 0,08 \\
\hline 67 & $\begin{array}{l}\text { Araucaria excelsa (Lamb.) R. } \\
\mathrm{Br} .\end{array}$ & Araucariaceae & - & 2 & 0,08 \\
\hline 68 & Chorisia speciosa A. St.-Hil. & Malvaceae & Paineira-rosa & 2 & 0,08 \\
\hline 69 & $\begin{array}{l}\text { Hibiscus pernambucensis } \\
\text { Arruda }\end{array}$ & Malvaceae & Algodão-da-praia & 2 & 0,08 \\
\hline 70 & Sterculia foetida $\mathrm{L}$. & Malvaceae & Chichá-fedorento & 1 & 0,04 \\
\hline 71 & Trema micrantha (L.) Blume & Cannabaceae & Pau-Pólvora & 1 & 0,04 \\
\hline 72 & $\begin{array}{l}\text { Enterolobium contortisiliquum } \\
\text { (Vell.) Morong }\end{array}$ & Fabaceae & Tamboril & 1 & 0,04 \\
\hline 73 & $\begin{array}{l}\text { Artocarpus altilis (Parkinson) } \\
\text { Fosberg }\end{array}$ & Moraceae & Fruta-pão & 1 & 0,04 \\
\hline 74 & Annona muricata $\mathrm{L}$. & Annonaceae & Graviola & 1 & 0,04 \\
\hline 75 & Dillenia indica $\mathrm{L}$. & Dilleniaceae & Árvore-da-pataca & 1 & 0,04 \\
\hline 76 & Lithrea molleoides (Vell.) Engl. & Anacardiaceae & Aroeira-branca & 1 & 0,04 \\
\hline 77 & $\begin{array}{l}\text { Peschiera fuchsiaefolia (A. DC.) } \\
\text { Miers }\end{array}$ & Apocynaceae & Leiteira & 1 & 0,04 \\
\hline 78 & $\begin{array}{l}\text { Anadenanthera macrocarpa } \\
\text { (Benth.) Brenan }\end{array}$ & Fabaceae & Angico & 1 & 0,04 \\
\hline 79 & Hymenaea courbaril L. & Fabaceae & Jatobá & 1 & 0,04 \\
\hline 80 & Ficus microcarpa L. f. & Moraceae & $\begin{array}{l}\text { Figueira- } \\
\text { lacerdinha }\end{array}$ & 1 & 0,04 \\
\hline
\end{tabular}




\begin{tabular}{|c|c|c|c|c|c|}
\hline $\mathbf{N}^{\mathbf{0}}$ & Espécies & Família & Nome popular & $\mathbf{P}$ & Pi (\%) \\
\hline 81 & Albizia julibrissin Durazz. & Fabaceae & $\begin{array}{l}\text { Acácia-de- } \\
\text { constantinópola }\end{array}$ & 1 & 0,04 \\
\hline 82 & $\begin{array}{l}\text { Senna macranthera (DC. ex } \\
\text { Collad.) H.S. Irwin \& Barneby }\end{array}$ & Fabaceae & Fedegoso & 1 & 0,04 \\
\hline \multirow[t]{2}{*}{83} & N.I. & - & - & 4 & 0,16 \\
\hline & & & Total & 2469 & 100,00 \\
\hline
\end{tabular}

A Tabela 2 mostra a riqueza das áreas de Salvador e de outros estudos no Brasil. Essa comparação deve levar em conta a diferença de amostragem, pois conforme aumenta o número de indivíduos amostrados, mais espécies são encontradas (Ricklefs, 2003).

Tabela 2 - Comparação da riqueza entre as áreas e outros estudos no Brasil $\left(\mathrm{N}-\mathrm{n}^{0}\right.$ indivíduos; R - Riqueza; R/N - densidade de espécies; MC - R. Miguel Calmon; DT - Dique do Tororó; CC - Corredor da Centenário; JMJ - Av. Juracy Magalhães Jr.; VG - Av. Vasco da Gama; ACM - Av. Antonio Carlos Magalhães; MLC - Macroárea Lucaia/Costeira).

Table 2 - Comparison of richness between areas and other studies in Brazil ( $\mathrm{N}$ - Number of individuals; R - Richness; R/N - Species density; MC - R. Miguel Calmon; DT - Dique do Tororó; CC - Corredor da Centenário; JMJ - Av. Juracy Magalhães Jr.; VG - Av. Vasco da Gama; ACM - Av. Antonio Carlos Magalhães; MLC - Lucaia/Costeira Macroarea).).

\begin{tabular}{lllll}
\hline \multicolumn{1}{c}{ Local } & $\mathbf{N}$ & $\mathbf{R}$ & $\mathbf{R} / \mathbf{N}$ & \multicolumn{1}{c}{ Referência } \\
\hline MC*$^{*}$ & 46 & 3 & 0,065 & - \\
DT $^{*}$ & 127 & 20 & 0,157 & - \\
Jacareí-SP & 295 & 28 & 0,095 & Faria; Monteiro; Fisch, 2007. \\
Pombal-PB & 212 & 8 & 0,038 & Rodolfo Júnior et al., 2008. \\
Campina Grande-PB & 360 & 24 & 0,067 & Araújo et al., 2009. \\
Taubaté/SP & 415 & 50 & 0,120 & Minhoto; Monteiro; Fisch, 2009. \\
Franca/SP & 479 & 72 & 0,150 & Silva et al., 2008. \\
CC $^{*}$ & 482 & 31 & 0,064 & - \\
JMJ $_{\text {VG* }}^{*}$ & 493 & 46 & 0,093 & - \\
ACM $^{*}$ & 500 & 53 & 0,106 & - \\
Campos de Jordão-SP & 821 & 44 & 0,054 & - \\
Norte do MT & 836 & 32 & 0,038 & Andrade, 2002. \\
UFBA* & 1210 & 37 & 0,031 & Almeida, 2009. \\
Assis/SP & 1723 & 103 & 0,060 & - \\
MLC* & 1915 & 54 & 0,028 & Rossatto; Tsuboy; Frei, 2008. \\
Americana-SP & 2469 & 82 & 0,033 & - \\
Maringá/PR & 2551 & 76 & 0,030 & Silva, 2005. \\
\hline
\end{tabular}

*áreas em Salvador-BA. 
Entre as áreas de Salvador a que apresentou maior número de espécies foi a UFBA (103), seguida das Avenidas Vasco da Gama (53) e Juracy Magalhães Jr. (43), contudo este fato sofre influência do grande número de indivíduos amostrados na UFBA (1723) e do diferencial desta como campus universitário (conforme citado anteriormente).

A densidade de espécies $(R / N)$ refere-se ao número de espécies encontradas em relação ao total de indivíduos (Odum, 1988). O Dique do Tororó foi o local com maior densidade em Salvador $(0,157)$, seguido da Avenida Vasco da Gama $(0,106)$ e da Juracy Magalhães $(0,093)$, mas esse número é favorecido pela baixa amostragem dessa área.

O Dique do Tororó apresentou densidade de espécies equiparável à encontrada por Silva (2008) em Franca-SP $(0,15)$ e um pouco superior a de Taubaté-SP $(0,12)$, estudada por Minhoto, Monteiro e Fisch (2009).

As Avenidas Vasco da Gama e Juracy Magalhães Jr. apresentaram uma riqueza maior do que a encontrada em áreas de Campos de Jordão-SP (Andrade, 2002) e norte do Mato Grosso (Almeida, 2009), mesmo considerando a maior amostragem destes estudos.

A riqueza de espécies amostradas na Macroárea Lucaia/Costeira em Salvador $(R=82)$ foi maior do que a encontrada por Silva (2005) em Americana-SP $(R=76)$ e consideravelmente elevada se comparado à de Maringá-PR $(R=87)$, conforme Blum, Borgo e Sampaio (2008), levando-se em conta o grande número de indivíduos amostrados nesta (93.261).

Quanto à diversidade, houve predomínio de T. catappa, C. fairchildiana e $P$. aquatica, que juntas representaram $28,38 \%$ da comunidade (Tabela 3). Das 82 espécies, as 14 mais abundantes responderam por $71,56 \%$ do total de indivíduos. Vinte espécies tiveram apenas uma ou duas ocorrências.

Tabela 3 - Percentual da soma das espécies mais abundantes na Macroárea Lucaia/Costeira.

Table 3 - Sum percentual of most abundant species in Lucaia/Costeira Macroarea.

\begin{tabular}{cc}
\hline $\mathbf{N}^{\circ}$ espécies & \% total \\
\hline 1 & 10,80 \\
2 & 20,48 \\
3 & 28,38 \\
4 & 33,89 \\
5 & 39,24 \\
6 & 44,06 \\
7 & 48,56 \\
8 & 52,85 \\
9 & 56,58 \\
10 & 60,06 \\
\hline
\end{tabular}


Para comparação com outras cidades brasileiras, será levada em conta a porcentagem do total de indivíduos representada pelas espécies mais abundantes, como consta na Tabela 3.

Algumas cidades brasileiras apresentam baixíssima diversidade, com dominância de poucas espécies. Rodolfo Júnior et al. (2008) na cidade de Pombal-PB, encontrou predomínio de $F$. benjamina e $S$. siamea que juntas foram responsáveis por aproximadamente $77 \%$ do total de indivíduos. No norte do Mato Grosso, Almeida (2009) encontrou que as três espécies mais frequentes representavam $79,1 \%$ do total de indivíduos, das cidades estudadas. Andrade (2002) encontrou que 87,8\% dos indivíduos arbóreos de Campos de Jordão pertenciam a duas espécies. Estes valores de dominância são muito superiores aos encontrados na capital baiana.

Na Macroárea, em Salvador, as oito espécies mais abundantes responderam por um pouco mais da metade (52,85\%) da comunidade arbórea. Em Jacareí-SP as quatro espécies mais freqüentes representaram em torno de 52\% dos indivíduos (Faria; Monteiro; Fisch, 2007), assim como em Taubaté-SP (Minhoto; Monteiro; Fisch, 2009). No bairro estudado por Araújo et al. (2009), em Campina Grande-PB, a espécie $F$. benjamina foi a mais freqüente $(51,95 \%)$ e as cinco mais abundantes totalizaram $83,62 \%$ da comunidade arbórea.

As dez espécies mais freqüentes, em Salvador-BA representaram 60,06\% do total da comunidade arbórea. Valor próximo ao de Franca-SP, 64,5\% (Silva et al., 2008). Em AssisSP, Rossatto, Tsuboy e Frei (2008) encontraram predomínio de L. tomentosa $(21,41 \%)$ e as dez espécies mais frequentes foram responsáveis por 82,77\% dos espécimes. Em Maringá$\mathrm{PR}$, as dez espécies mais frequentes responderam por $74,76 \%$ da arborização de vias públicas (Sampaio e De Angelis, 2008).

Em Águas de São Pedro-SP, ocorreu maior diversidade que em Salvador, pois a soma das dez espécies mais abundantes respondeu por $48,33 \%$ do total de indivíduos, apesar de C. peltophoroides apresentar freqüência de 13,63\% (Bortoleto et al., 2007).

Portanto, a Macroárea Lucaia/Costeira, em Salvador, apresenta considerável diversidade em comparação a outras cidades brasileiras, mas está longe do ideal, devido à dominância de poucas espécies e presença de muitas espécies com poucos indivíduos.

No total das áreas amostradas foram encontradas 27 famílias botânicas (Tabela 4), as quatro mais abundantes foram: Fabaceae $(\mathrm{Pi}=44 \%)$, Bignoniaceae $(\mathrm{Pi}=13,1 \%)$, Malvaceae $(\mathrm{Pi}=11 \%)$ e Combretaceae $(\mathrm{Pi}=10,8 \%)$. Merece destaque o fato de 
Combretaceae estar representada por apenas uma espécie, T. catappa. Famílias como Cannabaceae e Annonnaceae apresentaram apenas um indivíduo.

Tabela 4 - Famílias botânicas encontradas na Macroárea Lucaia/Costeira ( $\mathrm{P}$ - abundância; $\mathrm{Pi}$ - abundância relativa).

Table 4 - Botannical families found in Lucaia/Costeira Macroarea ( $P$ - Abundance; Pi Relative abundance).

\begin{tabular}{llll}
\hline Família & Espécies & P & Pi (\%) \\
\hline Fabaceae & 31 & 1085 & 44,00 \\
Bignoniaceae & 6 & 322 & 13,10 \\
Malvaceae & 7 & 272 & 11,00 \\
Combretaceae & 1 & 266 & 10,80 \\
Moraceae & 7 & 122 & 4,95 \\
Lythraceae & 1 & 92 & 3,73 \\
Anacardiaceae & 5 & 79 & 3,20 \\
Sapindaceae & 1 & 48 & 1,95 \\
Polygonaceae & 3 & 38 & 1,54 \\
Casuarinaceae & 1 & 34 & 1,38 \\
Cecropiaceae & 1 & 19 & 0,77 \\
Myrtaceae & 3 & 17 & 0,69 \\
Chrysobalanaceae & 1 & 17 & 0,69 \\
Lauraceae & 1 & 10 & 0,41 \\
Melastomataceae & 1 & 9 & 0,37 \\
\hline Outras & 12 & 39 & 1,56 \\
\hline Total & $\mathbf{8 2}$ & $\mathbf{2 4 6 9}$ & $\mathbf{1 0 0 , 0 0}$ \\
\hline
\end{tabular}

Faria, Monteiro e Fisch (2007) encontraram um maior número de espécies de Fabaceae e Bignoniaceae, em Jacareí-SP, sendo que em número de indivíduos predominaram as leguminosas. Bortoleto (2004) encontrou na Estância de Águas de São Pedro-SP, predomínio de Fabaceae, seguida de Bignoniaceae e Moraceae, considerando árvores, arbustos e palmeiras. Ou seja, assim como ocorre em Salvador, é comum a alta freqüência de leguminosas e bignoniáceas na arborização de ruas do país.

Houve predomínio de exóticas em relação ao número de espécies $(36,6 \%)$ e ao número de indivíduos, que representou um pouco mais da metade do total (53,3\%). O número de espécies nativas não regionais (21) foi um pouco inferior ao das nativas regionais (25), mas estas últimas representaram apenas 17,1\% do total de indivíduos (Tabela 5). 
Tabela 5 - Origem das espécies e abundância de indivíduos de cada categoria na Macroárea Lucaia/Costeira (NR - Nativa regional; BR - Nativa não regional; EX - Exótica; N.I. - Não identificado).

Table 5 - Species origin and individuals abundance of each category in Lucaia/Costeira Macroarea (NR - Regional native; BR - Non-regional native; EX - Exotic; N.I. - Not identified).

\begin{tabular}{lllll}
\hline Origem & $\mathbf{N}^{\circ}$ espécies & P espécies (\%) & $\mathbf{N}^{\circ}$ indivíduos & P indivíduos (\%) \\
\hline NR & 25 & 30,50 & 423 & 17,1 \\
BR & 21 & 25,60 & 651 & 26,4 \\
EX & 30 & 36,60 & 1316 & 53,3 \\
N.I. & 6 & 7,32 & 79 & 3,2 \\
\hline Total & $\mathbf{8 2}$ & $\mathbf{1 0 0 , 0 0}$ & $\mathbf{2 4 6 9}$ & $\mathbf{1 0 0 , 0}$ \\
\hline
\end{tabular}

Entre as nativas regionais, as mais freqüentes foram, em ordem crescente: $C$. peltophoroides (Sibipiruna); T. avellanedae (Ipê-rosa); C. ferrea (Pau-ferro); C. echinata (Pau-Brasil); S. mombin (Cajazeira); C. pachystachya (Embaúba); P. dubium (Canafístula); L. tomentosa (Oiti-mirim); S. terebinthifolia (Aroeira); T. granulosa (Quaresmeira); A. occidentale (Cajueiro); S. amara (Pau-Paraíba).

Muitos autores não fazem distinção entre as espécies nativas regionais e não regionais (Melo e Chagas, 2008; Faria, Monteiro e Fisch, 2007; Rossatto; Tsuboy; Frei, 2008). Estes estudos, portanto, subestimaram a presença de organismos exóticos ao bioma local.

Considerando-se a importância das variações regionais, dentro do mesmo país, muitos autores fazem a separação daquelas espécies do bioma local. Contudo, essa definição varia em virtude do território considerado. Silva (2008) encontrou em Franca-SP, apenas 8,3\% indivíduos nativos da região. Blum, Borgo e Sampaio (2008) encontraram 75,9\% das espécies exóticas ao ecossistema natural de Maringá.

Em Salvador ocorreu boa representatividade das espécies nativas regionais $(17,1 \%$ do total de indivíduos), em comparação a outras localidades do Brasil. Contudo deve-se levar em conta o amplo território considerado (Domínio da Mata Atlântica no estado da Bahia).

Das 82 espécies levantadas, apenas 30 fornecem alimento à fauna, o que representou $26,45 \%$ do total de indivíduos. Entre estas, as espécies mais abundantes foram, em ordem crescente: T. catappa (Amendoeira); $P$. aquatica (Monguba); $M$. indica (Mangueira); C. pachystachya (Embaúba); L. tomentosa (Oiti-mirim); S. cumini (Jamelão); P. 
americana (Abacateiro); P. ruscifolia (Algarobo); S. terebinthifolia (Aroeira); F. guaranitica (Figueira-branca).

Silva (2008) encontrou, em Franca-SP, 65\% dos indivíduos com potencial para alimentação de aves e morcegos, número bastante superior ao encontrado na capital baiana. Contudo, em Salvador não foi considerado o alimento para visitantes florais.

Quanto à ocorrência de espécies invasoras, foi detectada a presença das seguintes espécies arbóreas: $A$. heterophyllus (Jaqueira); $A$. indica (Nim); C. equisetifolia (Casuarina); L. leucocephala (Leucena); M. indica (Mangueira); M. caesalpiniifolia (Sabiá); T. catappa (Amendoeira). Além destas, as seguintes palmeiras e espécies arbustivas invasoras também estiveram presentes: Calotropis procera; Tecoma stans (Ipê-amarelo-de-jardim); Elaeis guineensis (Dendezeiro).

Em via pública de Pato Branco-PR, a espécie predominante, Ligustrum lucidum W. T. Aiton (alfeneiro, ligustro), também é invasora (Silva et al., 2007). Blum, Borgo e Sampaio (2008) levantaram 16 espécies invasoras em Maringá-PR, com destaque para Hovenia dulcis, Leucaena leucocephala, Melia azedarach e Tecoma stans.

Quanto à presença de plantas tóxicas ou com princípios alergênicos, foram encontradas as seguintes espécies arbustivas e arbóreas: Thevetia peruviana (Chapéu-deNapoleão); Nerium oleander (Espirradeira); Melia azedarach (Cinamomo); Schinus terebinthifolia (Aroeira); Lithrea molleoides (Aroeira-branca); Ficus microcarpa (Figueiralacerdinha).

Entre as plantas produzidas no Horto Municipal de Barreirinha (Curitiba-PR), 15 espécies apresentaram princípios tóxicos (Biondi e Leal, 2008). Cavalcanti et al. (2003) encontraram que 4,4\% das espécies arbóreas e 19\% dos outros vegetais ornamentais produzidos no Horto Florestal Lauro Pires Xavier, em Campina Grande-PB, apresentavam algum princípio tóxico.

Entre as alergênicas é importante comentar sobre a aroeira (S. therebinthifolia), uma espécie nativa regional, pioneira, medicinal, que proporciona alimento para a fauna, mas pode provocar alergia em pessoas sensíveis (Lorenzi, 2002; Lorenzi e Matos, 2000), portanto, o uso desta, em virtude de tantos benefícios, deve ser feito de forma criteriosa, em locais onde não haja fluxo constante de pessoas. 


\section{CONCLUSÕES}

Em virtude do exposto, conclui-se que na comunidade arbórea analisada em ruas e praças de Salvador-BA há:

1. grande riqueza de espécies, em comparação com outras localidades do Brasil;

2. dominância de poucas espécies, mas a diversidade mostrou-se maior que a de muitas cidades brasileiras;

3. predomínio de espécies e indivíduos exóticos e baixa representatividade de espécies nativas regionais;

4. baixa freqüência de espécies que oferecem alimento à fauna;

5. espécies potencialmente invasoras, tóxicas e alergênicas.

Além disso, percebeu-se que há um considerável predomínio de leguminosas (Fabaceae) e que a arborização dos campi da Universidade Federal da Bahia, em Salvador, diferencia-se do restante da cidade, em virtude da riqueza e composição específica.

\section{AGRADECIMENTOS}

Os autores agradecem aos membros do Jardim Botânico de Salvador, Parque Joventino Silva, Instituto de Permacultura da Bahia, Superintendência do Meio Ambiente e Superintendência de Conservação e Obras Públicas da Prefeitura Municipal de SalvadorBA, pelo apoio necessário para a realização deste trabalho, e a Luciano Souza dos Santos e Juliana Costa Piovesan pela revisão de inglês.

\section{REFERÊNCIAS BIBLIOGRÁFICAS}

ALMEIDA, D. N. de. Análise da arborização urbana de cinco cidades da região Norte do estado de Mato Grosso. 2009. 62 f. Dissertação (Mestrado em Ciências Florestais e Ambientais) - Curso de Pós-Graduação em Ciências Florestais e Ambientais, Faculdade de Engenharia Florestal, Universidade Federal de Mato Grosso, Cuiabá. 2009. 
ALMEIDA, W. R. de et al. Contextualização sobre Espécies Exóticas Invasoras: Dossiê Pernambuco. AMANE/TNC/CEPAN/CI-Brasil, Recife-PE. 2009.

ANDRADE, T. O. de. Inventário e análise da arborização viária da estância turística de Campos do Jordão, SP. 2002. 129 f. Dissertação (Mestrado em Agronomia) - Escola Superior de Agricultura Luiz de Queiroz, Universidade de São Paulo, Piracicaba. 2002.

APG II. An update of the Angiosperm Phylogeny Group classification for orders and families of flowering plants: APG II. Botanical Journal of the Linnean Society, UK, v. 141, p. 399-436, 2003.

ARAÚJO, A. C. de et al. Análise quali-quantitativa da arborização no Bairro Presidente Médici, Campina Grande-PB. Revista da Sociedade Brasileira de Arborização Urbana, Piracicaba - SP, v. 4, n. 1, p. 133-144, 2009.

BIONDI, D.; LEAL, L. Caracterização das Plantas Produzidas no Horto Municipal da Barreirinha - Curitiba / PR. Revista da Sociedade Brasileira de Arborização Urbana, Piracicaba-SP, v. 3, n. 2, p. 20-36, 2008.

BLUM, C. T.; BORGO, M.; SAMPAIO, A. C. F. Espécies exóticas invasoras na arborização de vias públicas de Maringá-PR. Revista da Sociedade Brasileira de Arborização Urbana, Piracicaba - SP, v. 3, n. 2, p.78-97, 2008.

BORTOLETO, S. Inventário quali-quantitativo da arborização viária da Estância de Águas de São Pedro-SP. 2004. 99 f. Dissertação (Mestrado em Fitotecnia) - Escola Superior de Agricultura Luiz de Queiroz, Universidade de São Paulo, Piracicaba. 2004.

BORTOLETO, S. et al. Composição e distribuição da arborização viária da Estância de Águas de São Pedro-SP. Revista da Sociedade Brasileira de Arborização Urbana, Piracicaba - SP, v. 2, n. 3, 2007. 
BRUN, F. G. K.; LINK, D.; BRUN, E. J. O emprego da arborização na manutenção da biodiversidade de fauna em áreas urbanas. Revista da Sociedade Brasileira de Arborização Urbana, Piracicaba - SP, v. 2, n. 1, 2007.

BRYANT, M. M. Urban landscape conservation and the role of ecological greenways at local and metropolitan scales. Landscape and Urban Planning, USA, v. 76, p. 23-44, 2006.

CARVALHO, G. M. de; ROQUE, N.; GUEDES, M. L. S. Levantamento das espécies arbóreas da Universidade Federal da Bahia, Salvador, Bahia. Sitientibus. Série Ciências Biológicas, Feira de Santana - BA, v. 7, n. 4, p. 377-387, 2007.

CAVALCANTI, M. L. F et al. Identificação dos vegetais tóxicos da cidade de Campina Grande-PB. Revista de Biologia e Ciências da Terra, Campina Grande - PB, v. 3, n. 1, 2003.

CHÃO VERDE Ltda. SPJ - Corredor da Centenário. SPJ/PMS. Salvador, BA. 2002.

CONDER - Companhia de Desenvolvimento Urbano do Estado da Bahia. Atlas do Desenvolvimento Humano da Região Metropolitana de Salvador. - Salvador: CONDER. PNUD. Fund. João Pinheiro, 2006.

FARIA, J. L. G.; MONTEIRO, E. A.; FISCH, S. T. V. Arborização de vias públicas do município de Jacareí - SP. Revista da Sociedade Brasileira de Arborização Urbana, Piracicaba - SP, v. 2, n. 4, p. 20-33, 2007.

HERRMANN, C. R. A. A qualidade do sombreamento de espécies arbóreas e sua influência no microclima do entorno imediato em ambientes urbanos. 2008. 160 f. Dissertação (Mestrado em Engenharia do Meio Ambiente) - Escola de Engenharia Civil, Universidade Federal de Goiás, Goiânia. 2008.

IBGE. Geografia do Brasil - Região Nordeste. Rio de Janeiro: SERGRAF, 1977. V. 2.

ISERNHAGEN, I.; BOURLEGAT, J. M. G. Le; CARBONI, M. Trazendo a riqueza arbórea regional para dentro das cidades: possibilidades, limitações e benefícios. Revista da Sociedade Brasileira de Arborização Urbana, Piracicaba - SP, v. 4, n. 2, p. 117-138, 2009. 
LONGA, C. M. O.; BARBOSA, M. do C. F.; OLIVEIRA, M. Z. A. de. Diagnóstico da vegetação da Rua Miguel Calmon. SUAVE/PMS. Salvador, BA. 1998.

LORENZI, H. Árvores Brasileiras - manual de identificação e cultivo de plantas arbóreas nativas do Brasil. 4 ed. Nova Odessa, SP: Instituto Plantarum, 2002.

LORENZI, H. et al. Árvores Exóticas no Brasil - madeireiras, ornamentais e aromáticas. Nova Odessa, SP: Instituto Plantarum, 2003. 384 p.

LORENZI, H. et al. Frutas brasileiras e exóticas cultivadas (de consumo in natura). São Paulo: Instituto Plantarum, 2006. 672 p.

LORENZI, H.; MATOS, F. J. A. Plantas Medicinais no Brasil: nativas e exóticas. Nova Odessa: Instituto Plantarum, 2000.

LORENZI, H; SOUZA, H. M. de. Plantas ornamentais no Brasil: arbustivas, herbáceas e trepadeiras. 3 ed. Nova Odessa, SP: Instituto Plantarum, 2001.

MALIARENKO, L.; BRITO, R. C. de. A introdução da vegetação de restinga na arborização urbana da Orla Marítima de Salvador. In: III Congresso Brasileiro de Arborização Urbana, 1996, Salvador - BA, Resumos...Salvador: SBAU/COELBA, 1996. 207 p.

MCPHERSON, G.; J. MUCHNICK . Effects of street tree shade on asphalt concrete pavement performance. Journal of Arboriculture, UK, v. 31, n. 6, 2005.

MELO, G. A. M. de; CHAGAS, E. P. Angiospermas utilizadas na arborização do Campus II da UNIFEOB, São João da Boa Vista, SP. Revista da Sociedade Brasileira de Arborização Urbana, Piracicaba - SP, v. 3, n. 4, p. 75-87, 2008.

MINHOTO, E. S.; MONTEIRO, E. A.; FISCH, S. T. V. Arborização viária na cidade de Taubaté, SP: no centro comercial histórico e um bairro Residencial Moderno. Revista da Sociedade Brasileira de Arborização Urbana, Piracicaba - SP, v. 4, n. 2, p. 82-96, 2009 
MMA (MINISTÉRIO DO MEIO AMBIENTE). Biodiversidade brasileira: avaliação e identificação de áreas e ações prioritárias para a conservação, utilização sustentável e repartição dos benefícios da biodiversidade nos biomas brasileiros. Secretaria de Biodiversidade e Florestas, MMA, Brasília, 2002.

ODUM, E. P. Ecologia. Rio de Janeiro: Editora Guanabara Koogan S.A. 1988.

OLIVEIRA, M. Z. A. de. Levantamento de pragas e fungos patogênicos como subsídio para o gerenciamento das áreas verdes de Salvador - Bahia. In: PEREIRA, T. S.; COSTA, M. L. M. N. da; JACKSON, P. W. (Orgs). Recuperando o verde para as cidades: a experiência dos Jardins Botânicos brasileiros. Rio de Janeiro: Instituto de Pesquisas Jardim Botânico do Rio de Janeiro. 2007.

OLIVEIRA, M. Z. A. de et al. Identificando espécies vegetais nativas, com potencial paisagístico, para arborização de áreas verdes da cidade de Salvador - Bahia. In: PEREIRA, T. S.; COSTA, M. L. M. N. da; JACKSON, P. W. (Orgs). Recuperando o verde para as cidades: a experiência dos Jardins Botânicos brasileiros. Rio de Janeiro: Instituto de Pesquisas Jardim Botânico do Rio de Janeiro. 2007.

OLIVEIRA, M. Z. A. de; NAKAGAWA, H.; FIGUEIREDO, L. A. Vistoria da Arborização do Dique do Tororó. GEROP/PMS. Salvador, BA. Julho, 2000.

PIVETTA, K. F. L.; SILVA FILHO, D. M. Arborização Urbana. Boletim Acadêmico. Série Arborização Urbana. Jaboticabal, SP: UNESP/FCAV/FUNEP, 2002.

PREFEITURA MUNICIPAL DE SALVADOR. Lei $\mathrm{n}^{\circ} 4.456$ de 09 de Dezembro de 1991. Disponível em: http://www.apms-ba.com.br/publicacao_view.jsp?idPub=539 . Acesso em: dez. 2009.

PREFEITURA MUNICIPAL DE SALVADOR. Lei $n^{\circ} 5.493$ de 19 de Janeiro de 1999. Disponível em: http://www.apms-ba.com.br/publicacao_view.jsp?idPub=547 . Acesso em: dez. 2009. 
RICKLEFS, R. E. A Economia da Natureza. 5 ed. Rio de Janeiro: Editora Guanabara Koogan S.A., 2003. 503 p.

RODOLFO JÚNIOR, F. et al. Análise da arborização urbana em bairros da cidade de Pombal no Estado da Paraíba. Revista da Sociedade Brasileira de Arborização Urbana, Piracicaba - SP, v. 3, n. 4, p. 3-19, 2008.

ROSSATTO D. R.; TSUBOY, M. S. F.; FREI, F. Arborização urbana na cidade de Assis-SP: uma abordagem quantitativa. Revista da Sociedade Brasileira de Arborização Urbana, Piracicaba - SP, v. 3, n. 3, p. 1-16, 2008.

SAMPAIO, A. C. F.; DE ANGELIS, B. L. Inventário e Análise da Arborização de Vias Públicas de Maringá-PR. Revista da Sociedade Brasileira de Arborização Urbana, Piracicaba - SP, v. 3, n. 1, p. 37-57, 2008.

SAVARD, J. P. L.; CleRgeAU, P.; MENNECHEZ, G. Biodiversity concepts and urban ecosystems. Landscape and Urban Planning, USA, v. 48, 2000.

SILVA, L. F. da. Situação da arborização viária e proposta de espécies para os bairros Antônio Zanaga I e II, da cidade de Americana/SP. 2005. 81 f. Dissertação (Mestrado em Agronomia) - Escola Superior de Agricultura Luiz de Queiroz, Universidade de São Paulo, Piracicaba. 2005.

SILVA, L. F. da. Interceptação da chuva nas espécies de Sibipiruna (Caesalpinia pluviosa DC.) e Tipuana (Tipuana tipu O. Kuntze). 2008. 61 f. Tese (Doutorado em Agronomia) Escola Superior de Agricultura Luiz de Queiroz, Universidade de São Paulo, Piracicaba. 2008.

SILVA, L. M. et al. Inventário e Sugestões para Arborização em via Pública de Pato Branco/PR. Revista da Sociedade Brasileira de Arborização Urbana, Piracicaba - SP, v. 2, n. 1, 2007.

SILVA, M. D. M. et al. Avaliação da Arborização de Vias Públicas de uma Área da Região Oeste da Cidade de Franca/SP. Revista da Sociedade Brasileira de Arborização Urbana, Piracicaba - SP, v. 3, n. 1, p. 19-35, 2008. 
SINITOX - Sistema Nacional de Informações Tóxico-Farmacológicas. Disponível em: http://www.fiocruz.br/sinitox . Acesso em: 12 de out. de 2009.

SOUZA, V. C.; LORENZI, H. Botânica sistemática: guia ilustrado para identificação das famílias de Angiospermas da flora brasileira, baseado em APG II. Nova Odessa, SP: Instituto Plantarum, 2005. 640 p.

SPANGENBERG, J. et al. Simulation of the Influence of Vegetation on Microclimate and Thermal Comfort in the City of São Paulo. Revista da Sociedade Brasileira de Arborização Urbana, Piracicaba - SP, v. 3, n. 2, p. 1-19, 2008.

SPJ. Caracterização de vias de circulação. Avenida Juracy Magalhães Júnior. SPJ/PMS. Salvador, BA. 2007.

SPJ. Caracterização de vias de circulação. Avenida Antonio Carlos Magalhães - Itaigara. SPJ/PMS. Salvador, BA. 2008a.

SPJ. Caracterização de vias de circulação. Avenida Vasco da Gama. SPJ/PMS. Salvador, BA. 2008b.

TAHA, H.; DOUGLAS S.; HANEY, J. Mesoscale meteorological and air quality impacts of increased urban albedo and vegetation. Energy and Buildings, USA, v. 25, 1997.

TROPICOS. Missouri Botanical Garden. Disponível em: http://www.tropicos.org. Acesso em: 17 dez. 2009.

VELASCO, G. del N. Potencial da arborização viária na redução do consumo de energia elétrica: definição de três áreas na cidade de São Paulo - SP, aplicação de questionários, levantamento de fatores ambientais e estimativa de Graus-Hora de calor. 2007. 13 f. Tese (Doutorado em Agronomia) - Escola Superior de Agricultura Luiz de Queiroz, Universidade de São Paulo, Piracicaba. 2007. 
WOLF, K. L. Freeway roadside management: the urban forest beyond the white line. Journal of Arboriculture, UK, v. 29, n. 3, 2003. 